\title{
Delay-Constrained Survivable Multicast Routing Problem on WDM Networks
}

\author{
Der-Rong Din and Jhong-Yan Jiang \\ Department of Computer Science and Information Engineering, \\ National Changhua University of Education, Changhua, Taiwan, R.O.C. \\ E-mail:deron@cc.ncue.edu.tw,m96612007@seed.net.tw \\ Tel: 886-4-7232105-7047 FAX: 886-4-7211284
}

\begin{abstract}
In WDM network, a link failure may cause service disruption and may lead to significant information loss. Especially, when the failed link is on a light-tree that carries traffic to multiple destinations, the traffic to all the downstream destinations along the failed link will be affected. Moreover, for a multicast request, the delay bound associated with it can be decided according to the emergence degree or priority of the data. Transmitted data with delay bound reflects the realistic demand in the future. For a given multicast request with delay bound, a delay-constrained survivable multicast protection provides the primary tree and a set of spare resources; when the single-link failure occurs, the pre-configured spare resources can be used to construct a new multicast tree under the delay constraint.

In this paper, given a multicast request with delay constraint, the goal is to allocate the delay-constrained primary multicast tree and protecting span p-cycles, when the single-link failure occurs, the multicast tree can be recovered by using protecting p-cycles such that the backup tree can also satisfy the delay constraint. This problem is defined as the Delay-Constrained Survivable Multicast Routing Problem (DCSMRP). The span pcycles protection scheme is studied in this paper and a heuristic method Delay Constrained Span p-cycles Protection (DCSP) is proposed to solve it. ${ }^{1}$
\end{abstract}

Keywords WDM, survivability, delay-constrained, multicast routing, p-cycles

\section{INTRODUCTION}

There is a growing consensus that the next generation Internet will employ an IP-over-WDM (wavelength-divisionmultiplexing) architecture. In order to provide various applications on WDM networks, mechanisms must be developed to handle not only point-to-point communications (or unicast) but also multicast. Multicast is the transmission of information from one source to multiple destinations simultaneously. Thus, issues concerning supporting multicast on WDM networks need to be studied [1]. For a comprehensive survey, the reader may refer to [1].

In WDM networks, a fiber can provide tremendous bandwidth, so a network failure (such as a fiber cut or node failure) can lead to a lot of light-paths failed and may cause enormous data loss. Therefore, several schemes have been proposed to achieve survivability by protection or restoration in the optical layer.

\footnotetext{
${ }^{1}$ This work was partly supported by the National Science Council (NSC) of Taiwan, R. O. C. under Grant Number NSC-98-2221-E-018-009.
}

To guarantee multicast traffic signals can be transmitted efficiently, transmission delay from the source to all destinations should be limited under a given delay bound. The delay bound of the multicast can be decided according to the emergence degree or priority of the data. For the WDM network with sparse light splitting and without wavelength conversion, several researchers [2] have studied the delayconstrained multicast routing problem for minimizing the number of fibers used or cost. The delay-constrained multicast routing problem in WDM networks is studied in [3], [4]. The goal is to minimize a linear combination of communication cost and wavelength consumption under delay constraint.

The light-tree [1] spanning all multicast members is used to establish point-to-multipoint connection for supporting multicast traffic. The fiber cut may cause service disruption and also may lead to heavy loss of significant information. For the failed link on a light-tree that carries traffic to multiple destinations, the traffic to all the downstream destinations along the failed link will be affected. The closer the link is to the source node, the more destinations will be affected [5].

Multicast protection schemes on WDM networks can be classified into five major protecting schemes: (1) tree-based protection [6]-[8]; (2) ring-based protection [9], [10]; (3) pathbased protection [11]-[13]; (4) segment-based protection [13], [14]; (5) cycle-based protection [5]. The most common method to protect light-trees for optical multicasting is to deploy a pair of link-disjoint or arc-disjoint trees (LDTs/ADTs) [7]. For the ring-based multicast protection approaches, Leelarusmee et al [10] proposed two methods: one ring for one multicast session (OFO) and one ring for all multicast sessions (OFA). For the optimal path-pair protection (OPP) approach, Singhal et al [13] reported an integer linear programming (ILP) model for protection of static multicast sessions. Moreover, Singhal et al also proposed OPP based-shared disjoint path (OPP-SDP) algorithm. In the OPP approach, a survivable multicast session is formed by all the link-disjoint primary and backup paths pairs from the source to every destination.

For unicast traffic protection, the pre-configuration protecting cycle (span p-cycles or p-cycles) [15] is a promising approach for protecting working capacity in WDM networks. The p-cycle provides two protection paths to recover the straddling span and a protection path to recover the on-cycle span [15]. Moreover, it can achieve ring-like recovery speed 
while maintaining the capacity efficiency of a mesh restorable network.

Recently, the goal of the studied multicast protection problems is to provide backup paths (or tree) to transmit the request. To the best of our knowledge, no related article takes the delay bound of multicast request into consideration. Given a delay-constrained multicast request, if a link on the multicast tree fails, the delay constraint of the backup paths (or tree) for the multicast request may not be satisfied. The problem is to find a multicast tree and a link-disjoint backup tree (paths or p-cycles) for a delay-constrained multicast request. When a link failure occurs, the pre-configured backup tree (paths or cycles) can be used to construct a new tree which satisfies the delay constraint.

In this paper, for a given network and a multicast request with delay bound, the goal is to find a primary multicast tree and the protecting p-cycles such that not only the primary multicast tree can satisfy the delay constraint, after recovering the multicast tree from single-link failure, the new tree can also satisfy the delay constraint. This problem is denoted as the Delay-Constrained Survivable Multicast Routing Problem $(D C S M R P)$. In this paper, the span p-cycles protection scheme used as the protecting scheme and the Delay Constrained Span p-cycles Protection (DCSP) algorithm is proposed to solve it.

\section{Problem DEFinition}

\section{A. Notations}

- $G(V, E)$ : the physical topology of the network, where $V$ is the set of nodes and $E$ is the set of links of $G$, respectively.

- $d_{e}$ : the propagation delay of link $e \in E$.

- $r=\{s, D, \Delta\}$; the multicast request, where $s \in V$ is the source node, $D=\left\{d_{1}, d_{2}, \ldots, d_{m}\right\}$ is the set of destinations, $m$ is the size of multicast request, $m=$ $|D| \leq|V|$, and $\Delta$ is the delay constraint of the request.

- $T_{r}$ : the primary multicast tree for the multicast request $r$.

- $K$ : a fixed positive integer.

- $\delta$ : the set of destinations which are sorted according to the delay of the destinations on $T_{r}$ in decreasing order.

- $P_{T_{r}}\left(s, d_{i}\right)$ : the path from $s$ to $d_{i}$ on $T_{r}, d_{i} \in D$ for the multicast request $r$.

- EPC: the set of existing span p-cycles on network.

- $E P C(e):$ the set of the span p-cycles in EPC $(E P C(e) \subseteq E P C)$, which can be used to protect the link $e \in E$ on either on-cycle or straddling way.

- $\overline{E P C(e)}: \overline{E P C(e)}=E P C \backslash E P C(e), e \in E$.

\section{B. Problem Definition}

For the multicast request $r$, after finding the primary multicast tree $T_{r}$, the delay of destination $d_{i}$ is the sum of delay of links on path $P_{T_{r}}$. That is, $\operatorname{delay}\left(d_{i}\right)=$ $\sum_{e \in P_{T_{r}}\left(s, d_{i}\right)} d_{e}, \forall d_{i} \in D$. The delay of the multicast tree, $\operatorname{delay}\left(T_{r}\right)$, is the maximum delay of all destinations and should be less than or equal to $\Delta$. That is, $\operatorname{delay}\left(T_{r}\right)=$ $\max _{\forall d_{i} \in D}\left\{\operatorname{delay}\left(d_{i}\right)\right\} \leq \Delta$.
In DCSMRP, given a WDM network $G(V, E)$ and a multicast request $r(s, D, \Delta)$, the goal is to find the primary multicast tree and the set of protecting cycles such that after recovering the multicast from the single-link failure, the delay of multicast tree can satisfy the delay constraint.

\section{Assumptions}

For the DCSMRP the following assumptions are given :

- For each link in $G$, there is a fiber which connects the end-nodes such that data can be transmitted bidirectional.

- The number of wavelengths of links are equal.

- All nodes in network are with full light-splitting and wavelength-converting capabilities.

- Only the single-fiber failure scenario is considered.

- The physical topology is two-connected.

- The number of transmitters/receivers of each node is fixed and known.

- Multicast requests are serialized such that a request is processed at a time.

\section{PROposed ALGORITHMS FOR MULTICAST ROUTING}

In this section, a method DCSP consisting of three heuristic algorithms are proposed to solve the DCSMRP. The proposed heuristic algorithms are Delay Constrained Span p-cycles Protection Algorithm (DCSPPA), Extending Delay Constrained pCycle Algorithm (EDCPCA), and Shortest Delay Path Cycle Finding Algorithm (SDPCFA). The details of the proposed algorithms are described in the following subsections.

\section{A. DCSPPA}

In this subsection, the Delay Constrained Span p-cycles Protection Algorithm (DCSPPA) is described. We assume that a set (EPC) of span p-cycles is already constructed and used to protect the primary multicast tree of multicast request. For the arrived multicast request, the goal is to find a primary multicast tree $\left(T_{r}\right)$ and a set of protecting cycles to protect the multicast. That is, for each edge of the primary multicast tree, there is a p-cycle in either on-cycle or straddling manner to protect the edge.

In general, a multicast traffic is unlikely to be symmetric in both directions between any two nodes. This implies that the number of working and protection wavelengths is not likely to be the same in both directions on a link. Therefore, without loss of generality, all trees and p-cycles considered in this paper are unidirectional, i.e., they are directed. The existing cycle can be used if there are enough capacities and can satisfy the delay constraint, otherwise a new protecting cycle is found to protect the edge. The Extending Delay Constrained p-Cycle Algorithm (EDCPCA) is proposed to find a cycle by extending existing cycles. If performing EDCPCA fails to find a cycle, then the Shortest Delay Path Cycle Finding Algorithm (SDPCFA) is used to find a whole new one.

Since the delay of the cycle and the path may affect the delay of the new multicast tree, to recover multicast tree such that the delay constraint can be satisfied, these delays should be talked into consideration. The delay of a p-cycle $C$ is the 


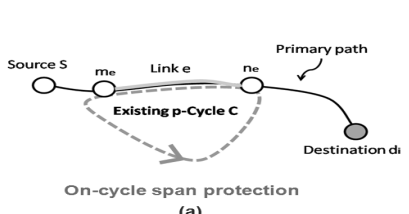

(a)

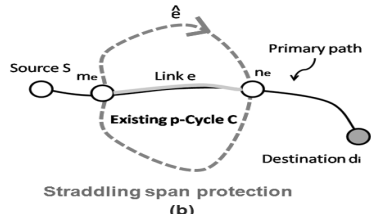

Fig. 1. Delay of backup path (a) on-cycle span, (b) straddling span.

sum of the delays of all edges and denoted as $\operatorname{delay}(C)=$ $\sum_{e \in C} d_{e}$. If the edge is an on-cycle span, as the case showed in Fig.1(a). The counter-clockwise cycle can be used to protect the edge under the delay constraint if the formula (1) can be satisfied.

$$
\operatorname{delay}(C)+\operatorname{delay}\left(d_{i}\right)-2 \times d_{e} \leq \Delta, e \in T_{r}
$$

It is worth noting that, not all cycles can be used to protect the on-cycle span due to the cycle is unidirectional.

If the edge is a straddling span of the protecting cycle, as the illustration showed in Fig.1(b). The cycle can be used to protect the edge under the delay constraint if the formula (2) can be satisfied,

$$
\operatorname{delay}(\hat{e})+\operatorname{delay}\left(d_{i}\right)-d_{e} \leq \Delta, e \in T_{r},
$$

where $\operatorname{delay}(\hat{e})$ is the delay of the default backup path on cycle.

First, let the initial value of the integral variable $k$ be zero, the Dijkstra-based least-delay tree algorithm (LDTA) is used to find the primary multicast tree $T_{r}^{0}$. If delay-constrained tree $T_{r}^{0}$ cannot be found, the request is blocked and return.

At the $k^{\text {th }}$ iteration, for the current primary multicast tree $T_{r}^{k}(0 \leq k \leq K)$, if the protecting cycles cannot be found for all edges in $T_{r}^{k}$, then another primary tree $T_{r}^{k+1}$ whose delay is slightly larger than current one is used as the new primary multicast tree. Then another set of protecting cycles are found to protect all edges on new tree $T_{r}^{k+1}$. To find a new multicast tree, the $k^{t h}(1 \leq k \leq K \leq n-1)$ largest delay edge of the primary tree $T_{r}^{0}$ is removed from the topology $G$ and form $G^{k}$, then LDTA is performed to find a multicast tree $T_{r}^{k}$ under delay constraint on $G^{k}$. If $T_{r}^{k}$ is found, then find a set cycles to protect all edges on tree $T_{r}^{k}$. If all edges can be protected by the set of cycles under delay constraint then return. Otherwise, if $k<K$, increase $k$ by one and repeat the process to find a new tree $T_{r}^{k}$ and protecting cycles. If trees $T_{r}^{k}$ and cycles cannot be found in all the cases $0 \leq k \leq K$, the multicast request is blocked. The details of the DCSPPA is known as follows.

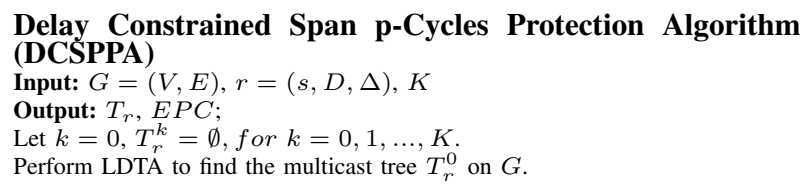

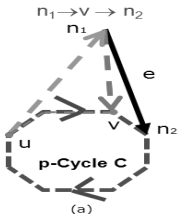
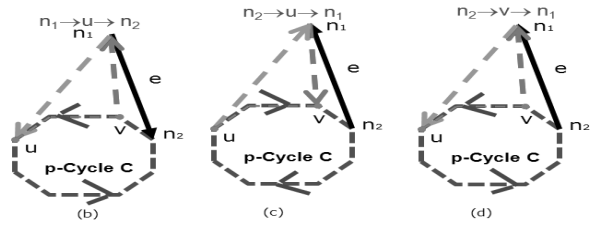

Fig. 2. EDCPC example (a) case 1, (b) case 2 (c) case 3, (d) case 4.

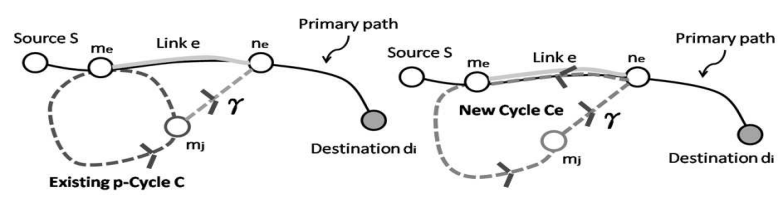

(a)

(b)

Fig. 3. EDCPC algorithm illustration (a) before, (b) after.

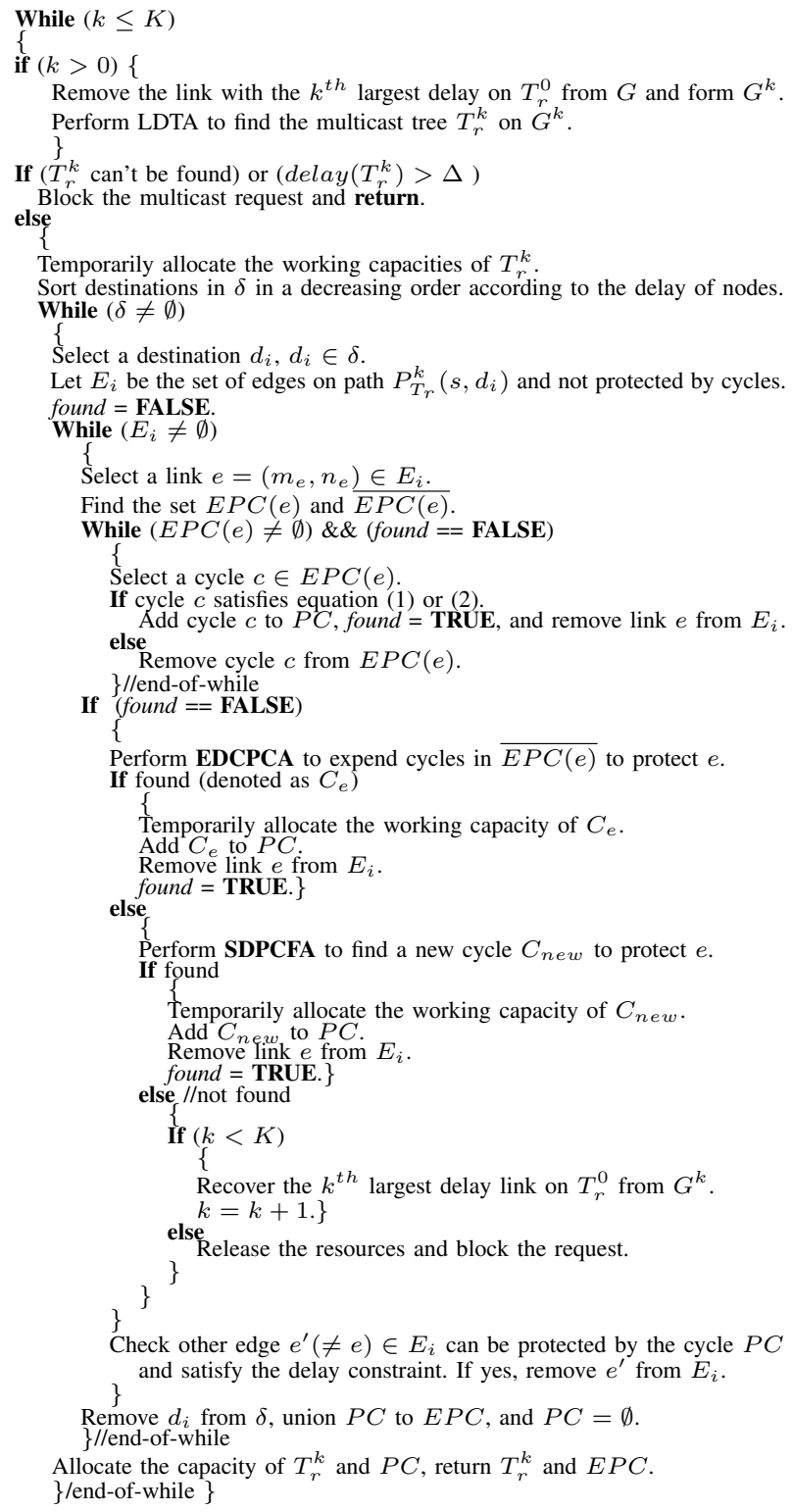




\section{B. $E D C P C A$}

Four cases are considered in performing the EDCPCA. In these cases (see Fig. 2), for a directed edge $e=\left(n_{1}, n_{2}\right)$, a cycle $c \in \overline{E P C(e)}$ which passes through end node $n_{1}$ (or $\left.n_{2}\right)$ is selected and to be extended to protect edge $\left(n_{1}, n_{2}\right)$. According to the relation between $e$ and the selected cycle $c$, the LDTA is performed to find two link-disjoint paths from $n_{1}$ to $u$ and $v$ to $n_{1}(u \neq v)$, (nodes $u$ and $v$ are on the cycle $c$ ). Then, the path between $u$ and $v$ of the cycle is removed and paths $u$ to $n_{1}, n_{1}$ to $v$, and cycle $c$ are joined to form a protecting cycle for $e$.

The details of the EDCPCA is showed as follows and the example is shown in Fig. 3.

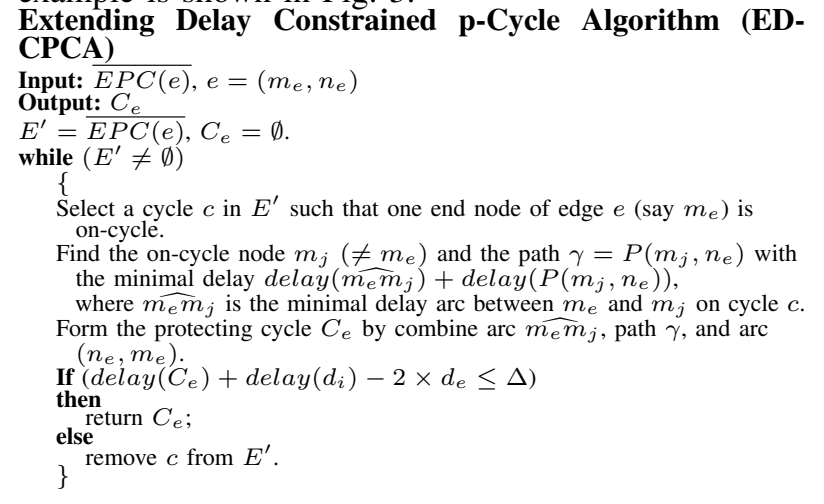

\section{SDPCFA}

If performing EDCPCA fails to extend existing cycles to protect the edge $e$, the Shortest Delay Path Cycle Finding Algorithm (SDPCFA) is performed to find a new cycle under delay constraint to protect edge $e$ in on-cycle manner. The details of the SDPCFA is shown as follows and the example of SDPCFA is shown in Fig. 4

\section{Shortest Delay Path Cycle Finding Algorithm (SDPCFA)}

Input: $G_{r}, e=\left(m_{e}, n_{e}\right)$

Construct auxiliary graph $G_{i}^{e}$ by removing $e$ from $G_{r}$

Use Shortest Delay Path algorithm to find backup path $B_{G_{i}}\left(m_{e}, n_{e}\right)$ for link

$e$ on graph $G_{i}^{e}$, where delay $\left(B_{G_{i}^{e}}\left(m_{e}, n_{e}\right)\right)+\operatorname{delay}\left(d_{i}\right)-d_{e} \leq \Delta$.

If $B_{G_{i}^{e}}\left(m_{e}, n_{e}\right)$ is found, join $\operatorname{arc}\left(n_{e}, m_{e}\right)$ and path $B_{G_{i}^{e}}\left(m_{e}, n_{e}\right)$ to form a new cycle $C_{n e w}$.

return $C_{n e w}$ ew
else return false.

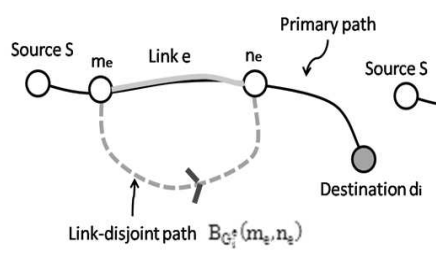

(a)

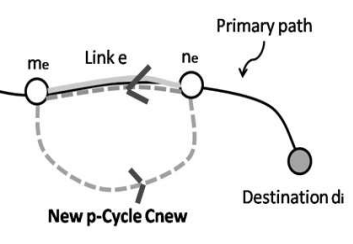

(b)
Fig. 4. SDPCFA illustration (a)before, (b) after.

\section{EXPERIMENTAL Results}

To know the efficiency of the proposed algorithms, three criteria are used and evaluated through experiments. They are blocking ratio (BR), resource utility ratio (RUR), and wavelength efficient ratio (WER). BR is the probability that the primary multicast tree and protecting cycles cannot be found with delay constraint. RUR is the ratio of the spare capacity to that of the working capacity. WER is the ratio of the working capacity to total (working and spare) capacity.

The USNET topology (as shown in Fig.5(a)) with 24 nodes and 43 links was used for simulations. The delay of link in USNET is proportional to the length of the link. The proposed algorithms are conducted by $\mathrm{C}++$ language together with the BGL (Boost Graph Library) [16]. All simulations were run on a PC with Core duo $1.66 \mathrm{GHz}$ CPU, 1.5GB RAM, and Windows XP operating system. In each simulation, multicast requests are generated randomly, the arrival rate of requests are fitted with the Poisson distribution with mean 3 . The delay bound of multicast request is set between 5.0 and 9.0, and the value of $K$ is in $\{0,5,8,10\}$. For each multicast request, the size of $D$ is randomly generated and within range 2-11 and the source node and the destinations are randomly selected.

\section{A. Efficiency of DCSP}

First, RUR and BR are evaluated for cases that with $R$ (= 30 or 40 ) multicast requests, $W$ (=16 or 32 ) wavelengths, and for different values of the $\Delta$; the results are shown in Fig.6. The result in Fig.6(a) shows that the value of RUR increases as the value of $\Delta$ decreases. This is may be the reason that the tighter of delay-constraint, the harder existing p-cycles can be used to protect the multicast requests. Thus, new p-cycles with smaller delay should be found and this may increase the RUR value. This appears on the cases for different numbers of multicast requests, the RUR value of $R=40$ is better than that of $R=30$. The RUR related to the different numbers of wavelengths provided by the WDM network is unapparent.

The result in Fig.6(b) shows the value of BR increases as the value of $\Delta$ decreases. The BR value of case $R=40$ is worse than that of the case $R=30$ for $\Delta<7.0$. But for $\Delta>7.0$, the BR values of two cases are very close. The BR value of $W=16$ is worse than that of $W=32$.

The relations of $\mathrm{BR}$ value, computational time, and the number of multicasts are shown in Fig.7. The value of BR decreases as the number of multicast requests increases (as shown in Fig.7(a)). This may the reason that the span pcycles reserved by previous multicast requests can be shared and used to protect some new follow-up multicasts. Fig.7(a) also shows the results for different values of $K$, BR value can be reduced as $K$ decreases. The difference of BR values of cases $K=0$ and $K=10$ is about $10 \%$. Fig.7(b) shows that the computational time is proportional to the number of requests. Moreover, the computational time also increases as $K$ increases.

\section{B. Comparison}

Two methods proposed in [17] were used for comparisons. They are DCLTP (Delay-constrained Link-disjoint Trees Protection) and DCDTP (Delay-constrained Dual-tree Protection). For the DCLTP, link-disjoint primary and backup trees with delay-constraint are found and used to protect the multicast 
request. For the DCDTP, a primary multicast tree and a bidirectional path which connected source to all destinations on the leaves of primary multicast tree are used to protect the multicast request.

The results (in Fig.5(b)) show that the BR value of DCDTP rapidly increases from $25 \%$ to $69 \%$ as the number of requests increases, and is worse than that of DCSP (30\%-40\%). Thus BR value provided by the DCSP method is comparatively stable and better than that of the DCLTP. The computational complexity of the DCLTP, DCDTP, and DCSP is $O\left(K|V|^{3}\right)$, $O\left((K+|D|)|V|^{3}\right)$, and $O\left((K+|D|+2)|V|^{3}\right)$, respectively. The result in Fig.8(a) shows that RUR value increases as the $\Delta$ decreases for $R=40$. The RUR and WER of DCSP values are better than that of DCLTP and DCDTP in the cases of $\Delta<7.0$ (as shown in Fig.8).

\section{Conclusions}

In this paper, we define and study the Delay-Constrained Survivable Multicast Routing Problem (DCSMRP). The span p-cycles protection scheme is used and a heuristic algorithm Delay Constrained Span p-cycles Protection (DCSP) method is proposed to solve it. Simulations show that the value of BR of DCSP is worse than DCDTP but better than DCLTP when network with enough wavelength resources.
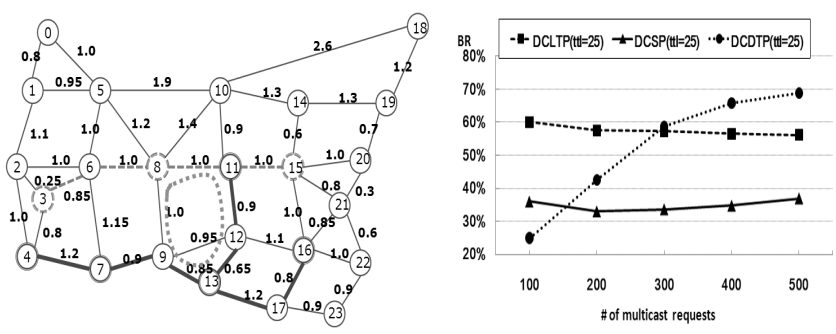

Fig. 5. (a) USNET, (b) comparison of BR for DCLTP, DCDTP and DCSP with $\mathrm{TTL}=25$.
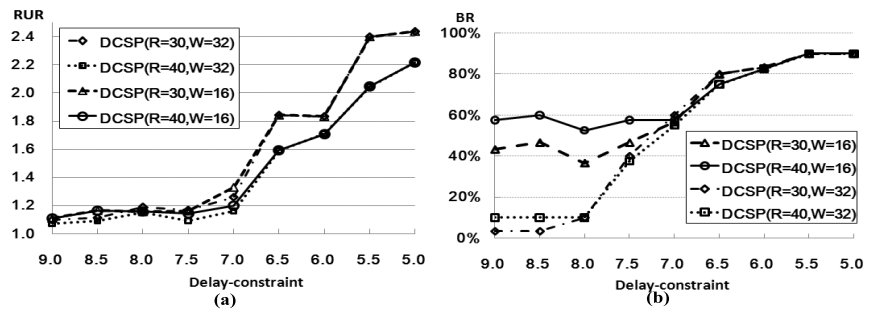

Fig. 6. Simulation results (a) RUR, (b) BR.
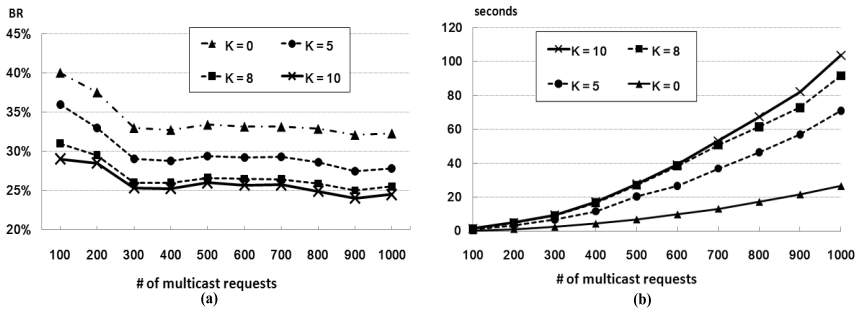

Fig. 7. Simulation results (a) $K$, (b) CPU time.

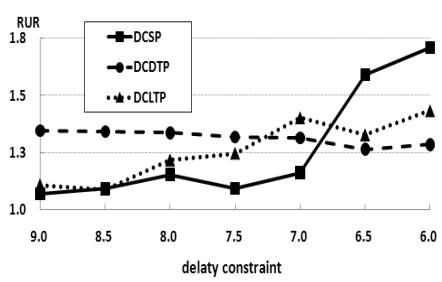

(a)

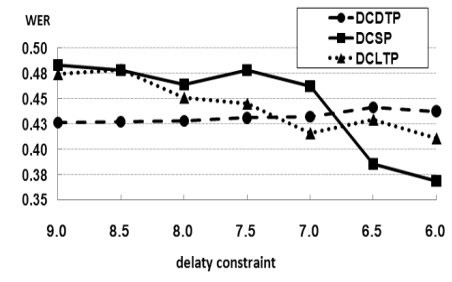

(b)
Fig. 8. Comparison DCLTP, DCDTP and DCSP (a)RUR, (b) WER

\section{REFERENCES}

[1] Biswanath Mukherjee, Optical WDM Networks, Springer press, 2006.

[2] A. Yan, M. Ali, J. Deogun, "Routing optimization of multicast sessions in sparse light-splitting optical networks," in Proc. of IEEE GLOBECOM 2001, vol.4, pp.2134-2138.

[3] DR Din, "Heuristic algorithms for finding light-forest of multicast routing on WDM network," JISE, vol. 25, no.1, pp.83-103, 2009.

[4] DR Din, "Genetic algorithm for finding minimal cost light-forest of multicast routing on WDM network," Artificial Intelligence Review.

[5] F. Zhang, WD Zhong, and Y. Jin, "Optimizations of p-cycle-based protection of optical multicast sessions," J. Lightw. Technol., vol. 26, no. 19, pp.3298-3306, 2008

[6] N.K. Singhal, C. Ou, and B. Mukherjee, "Cross-sharing vs. self-sharing trees for protecting multicast sessions in mesh networks," Comput. Netw., vol. 50, no. 2, pp.200-206, 2006

[7] N. K. Singhal and B. Mukherjee, "Protecting multicast sessions in WDM optical mesh networks," J. Lightw. Tech., 21(4), pp.884-892, 2003.

[8] M. Medard, S. G. Finn, R. A. Barry, and R. G. Gallager, "Redundant trees for preplanned recovery in arbitrary vertex-redundant or edgeredundant graphs," IEEE/ACMTrans. Netw., 7(5), pp.641-652, 1999.

[9] T. Rahman and G. Ellinas, "Protection of multicast sessions in WDM mesh optical networks," in Proc. of OFC'05, Mar. 2005, p. 3.

[10] P. Leelarusmee, C. Boworntummarat, and L. Wuttisittikulkij, "Design and analysis of five protection schemes for preplanned recovery in multicast WDM networks," in Proc. of IEEE SAWWC'04, Apr.2004, pp.167-170.

[11] H. B. Luo, H. F. Yu, L. M. Li, and S. Wang, "On protecting dynamic multicast sessions in survivable mesh WDM networks," in Proc. of OFC'06, Anaheim, CA, Mar. 2006, p. 3.

[12] N. K. Singhal and B. Mukhejee, "Dynamic provisioning of survivable multicast sessions in optical WDM mesh networks," in Proc. of Optical Fiber Communications Conf., Mar. 2003, pp.207-209.

[13] N. K. Singhal, L. H. Sahasrabuddhe, and B. Mukherjee, "Provisioning of survivable multicast sessions against single link failures in optical WDM mesh networks," J. Lightw. Technol., 21(11), pp.2587-2594, 2003.

[14] C. Lu, H. Luo, S. Wang, and L. M. Li, "A novel shared segment protection algorithm for multicast sessions in mesh WDM networks," ETRI J., 28, pp.329-336, 2006.

[15] W. D. Grover and D. Stamatelakis, "Cycle-oriented distributed preconfiguration: Ring-like speed with mesh-like capacity for self-planning network restoration," in Proc. of ICC'98, Nov. 1998, vol. 1, pp. 537-543.

[16] http://www.boost.org/doc/libs/1_41_0/libs/graph/doc/index.html

[17] JY Jiang, A study of Delay-constrained survivable multicast routing on WDM networks, Mastrer Thesis, 2009, National Changhua University of Education. 